\title{
Climbing three-Reggeon ladders: Four-loop amplitudes in the high-energy limit in full color
}

\author{
Giulio Falcioni $\odot,{ }^{1, *}$ Einan Gardi, ${ }^{1, \dagger}$ Calum Milloy $\odot,{ }^{2, \star}$ and Leonardo Vernazza ${ }^{2, \S}$ \\ ${ }^{1}$ Higgs Centre for Theoretical Physics, School of Physics and Astronomy, The University of Edinburgh, \\ Edinburgh EH9 3FD, Scotland, United Kingdom \\ ${ }^{2}$ Dipartimento di Fisica and Arnold-Regge Center, Universitá di Torino, and INFN, Sezione di Torino, \\ Via P. Giuria 1, I-10125 Torino, Italy
}

(Received 10 December 2020; accepted 10 May 2021; published 7 June 2021)

\begin{abstract}
We show that an iterative solution of rapidity evolution equations with a leading-order kernel renders the entire signature-odd tower of next-to-next-to-leading logarithmic (NNLL) contributions to partonic $2 \rightarrow 2$ amplitudes in the Regge limit computable in full color. The result, which universally holds in any gauge theory, corresponds to a set of three-Reggeon ladder diagrams. In this setting we compute the NNLL amplitude to four loops in dimensional regularization through finite corrections. Furthermore, by contrasting the result with the exponentiation properties of soft singularities we determine the four-loop correction to the soft anomalous dimension at this logarithmic accuracy. The latter features quartic Casimir contributions beyond those appearing in the cusp anomalous dimension. Finally, in the case of $\mathcal{N}=4$ super Yang-Mills, we also determine the finite hard function at four loops through NNLL in full color.
\end{abstract}

DOI: 10.1103/PhysRevD.103.L111501

\section{INTRODUCTION}

The high-energy limit of gauge-theory scattering amplitudes has long been a source of unique insight into gauge dynamics. Amplitudes drastically simplify in this limit, and their factorization in rapidity reveals new degrees of freedom that propagate in two transverse dimensions. Rapidity evolution equations, Balitsky-Fadin-Kuraev-Lipatov (BFKL) $[1,2]$ and its nonlinear generalization [3], manifest concepts from Regge theory [4], leading to remarkable new insights. Several avenues have emerged in recent years which exploit the predictive power of factorization and evolution in rapidity, and translate it into concrete predictions for partonic amplitudes [5-13]. New results regarding the Regge limit have been instrumental in determining multi-leg planar $\mathcal{N}=4$ super Yang-Mills amplitudes in general kinematics to unprecedented accuracy, see e.g., $[6,14,15]$. In parallel, computation of the Regge limit in $2 \rightarrow 2$ scattering for general color [7-10] have been shown to provide powerful constraints on soft singularities of amplitudes in general kinematics [11,16-18].

\footnotetext{
*Giulio.Falcioni@ed.ac.uk

†Einan.Gardi@ed.ac.uk

CalumWilliam.Milloy@unito.it

${ }^{\S}$ Leonardo.Vernazza@to.infn.it
}

Published by the American Physical Society under the terms of the Creative Commons Attribution 4.0 International license. Further distribution of this work must maintain attribution to the author(s) and the published article's title, journal citation, and DOI. Funded by SCOAP.
Our focus here is on universal features of $2 \rightarrow 2$ gaugetheory scattering amplitudes, $1+2 \rightarrow 3+4$, in the highenergy limit, following [7-11]. These amplitudes are described by two independent Mandelstam invariants $s \equiv$ $\left(p_{1}+p_{2}\right)^{2}$ and $t \equiv\left(p_{1}-p_{4}\right)^{2}$, with $u \equiv\left(p_{1}-p_{3}\right)^{2}=$ $-s-t$, where in the high-energy limit $s \gg-t$, and we apply perturbation theory, assuming that the momentum transfer $-t$ is large compared to the QCD scale. For $s \gg-t$ the perturbative amplitude is dominated by large logarithms in the ratio $\frac{s}{-t}$. Famously, the leading logarithms (LL) can be resummed to all orders $[19,20]$ via

$$
\mathcal{M}_{i j \rightarrow i j}^{\mathrm{LL}}(s, t)=\left(\frac{s}{-t}\right)^{C_{A} \alpha_{g}(t)} \mathcal{M}_{i j \rightarrow i j}^{\mathrm{tree}}
$$

where $\mathcal{M}_{i j \rightarrow i j}^{\text {tree }}=g_{s}^{2} \frac{2 s}{t} \mathbf{T}_{i} \cdot \mathbf{T}_{j}$ is the tree-level amplitude with the generator $\mathbf{T}_{i}$ in the representation of parton $i$, and

$$
\alpha_{g}=\frac{\alpha_{s}}{\pi} \frac{r_{\Gamma}}{2 \epsilon}+\mathcal{O}\left(\alpha_{s}^{2}\right) ; \quad r_{\Gamma}=e^{\epsilon \gamma_{E}} \frac{\Gamma^{2}(1-\epsilon) \Gamma(1+\epsilon)}{\Gamma(1-2 \epsilon)}
$$

is the gluon Regge trajectory, presented here in dimensional regularization with $D=4-2 \epsilon$, where we suppressed higher-order corrections which contribute beyond LL. The simple exponentiation property in (1), with the characteristic color charge $C_{A}$, can be understood as due to the exchange of a single Reggeized gluon (dubbed Reggeon), which admits a trivial evolution equation in rapidity. At higher logarithmic accuracy more complex 
analytic structure emerges, associated with compound states of multiple Reggeons [21-23]. While the corresponding evolution equations are integrable in the planar limit [24-27], they are difficult to solve in general. Nevertheless, they can be integrated iteratively [7-10] to obtain perturbative high-energy amplitudes order-by-order in $\alpha_{s}$. In this paper we extend these methods to the entire tower of nextto-next-to-leading logarithmics (NNLLs), and perform explicit computations to four loops.

In the Regge limit amplitudes naturally split according to their signature symmetry; $\mathcal{M}=\mathcal{M}^{(-)}+\mathcal{M}^{(+)}$where $\mathcal{M}^{(-)}$and $\mathcal{M}^{(+)}$are respectively odd and even under $s \leftrightarrow u$. Upon using a signature-symmetric definition for the large logarithm,

$\frac{1}{2}\left(\log \frac{-s-i 0}{-t}+\log \frac{-u-i 0}{-t}\right)=\log \left|\frac{s}{t}\right|-i \frac{\pi}{2} \equiv L$,

and expanding $\mathcal{M}^{(-)}$and $\mathcal{M}^{(+)}$according to

$$
\mathcal{M}_{i j \rightarrow i j}^{( \pm)}=\sum_{n=0}^{\infty}\left(\frac{\alpha_{s}}{\pi}\right)^{n} \sum_{m=0}^{n} L^{m} \mathcal{M}_{i j \rightarrow i j}^{( \pm, n, m)}
$$

with $\mathcal{M}_{i j \rightarrow i j}^{(-, 0,0)} \equiv \mathcal{M}_{i j \rightarrow i j}^{\text {tree }}$, it can be shown [8] that the odd amplitude coefficients $\mathcal{M}_{i j \rightarrow i j}^{(-, n, m)}$ are purely real, while the even ones $\mathcal{M}_{i j \rightarrow i j}^{(+, n)}$ are purely imaginary. Since Bose symmetry links the kinematic dependence to that of color, $\mathcal{M}^{(+)}$and $\mathcal{M}^{(-)}$are governed by $t$-channel exchange of color representations which are respectively even and odd under $1 \leftrightarrow 4$ (or $2 \leftrightarrow 3$ ) interchange. The latter consist of an odd number of Reggeons, while the former an even number [7]. Signature is preserved under rapidity evolution, and this greatly simplifies the computation of these amplitudes [7-10]. The signature even amplitude $\mathcal{M}^{(+)}$, which starts at next-to-leading logarithmic (NLL) accuracy is governed at this logarithmic order by two-Reggeon exchange, satisfying BFKL evolution. Using an iterative solution of this equation, this entire tower of logarithms has recently been determined $[7,9,10]$.

The present paper focuses on the signature odd amplitude. At NLL accuracy, $\mathcal{M}_{i j \rightarrow i j}^{(-)}$is still governed by a single Reggeon exchange (Regge pole) [28], with $\mathcal{O}\left(\alpha_{s}^{2}\right)$ corrections to the trajectory in (2) [29] along with $s$-independent impact factors [8]:

$\mathcal{M}_{i j \rightarrow i j}^{(-), \mathrm{LL}+\mathrm{NLL}}=e^{C_{A} \alpha_{g}(t) L} Z_{i}(t) D_{i}(t) Z_{j}(t) D_{j}(t) \mathcal{M}_{i j \rightarrow i j}^{\text {tree }}$.

The factors $Z_{i}(t)$ and $D_{i}(t)$ admit perturbative expansions with coefficients $Z_{i}^{(n)}$ and $D_{i}^{(n)}$ where the one-loop terms, $n=1$, contribute at NLL, while the higher-order terms give rise to further subleading logarithms. $Z_{i}(t)$ generate collinear singularities [16]

$$
Z_{i}(t)=\exp \left\{-\frac{1}{2} \int_{0}^{\mu^{2}} \frac{d \lambda^{2}}{\lambda^{2}} \Gamma_{i}\left(\alpha_{s}\left(\lambda^{2}\right), \lambda^{2}\right)\right\}
$$

with $\Gamma_{i}=\frac{1}{2} \gamma_{K}\left(\alpha_{s}\left(\lambda^{2}\right)\right) C_{i} \log \frac{-t}{\lambda^{2}}+2 \gamma_{i}$. Here $\gamma_{K}$ is the universal cusp anomalous dimension [30-32], $C_{i}$ is the quadratic Casimir in the representation of parton $i$ and $\gamma_{i}$ are anomalous dimensions associated with on-shell form factors [33,34]. The collinear-subtracted impact factors $D_{i}(t)$ are known to two loops [8].

Our new computation concerns the NNLL tower, which manifests a Regge cut in the real part of the amplitude [7,34-37]. This phenomenon is associated with the exchange of three Reggeons, as shown by direct calculations to three loops $[8,37]$. Here we take a further step by showing that the entire NNLL tower, $\mathcal{M}^{(-, n, n-2)}$, can be computed using the evolution of one and three Reggeons and the transitions between them. We then explicitly compute the NNLL amplitude at four loops in full color. Finally, upon comparing the result with the known exponentiation properties of infrared singularities we gain a powerful check and determine the soft anomalous dimension in the same approximation.

\section{METHODOLOGY}

We describe $2 \rightarrow 2$ scattering at high energy following [7]. Fast particles moving in the plus $(+)$ lightcone direction appear as infinite Wilson lines [38]

$$
e^{i g_{s} \mathbf{T}^{a} W^{a}(z)} \equiv \mathcal{P} \exp \left[i g_{s} \mathbf{T}^{a} \int_{-\infty}^{+\infty} d x^{+} A_{+}^{a}\left(x^{+}, x^{-}=0, z\right)\right]
$$

at transverse position $x_{\perp}=z$, where the generator $\mathbf{T}^{a}$ is in the representation of the scattering parton and $W^{a}(z)$ identifies a Reggeon field. Rapidity divergences are regulated by introducing a cutoff $\eta=L$. The projectile and target in the scattering process, denoted respectively as $\left|\psi_{i}\right\rangle$ and $\left\langle\psi_{j}\right|$, are expanded in Reggeon fields, regulated at different rapidities. These are then evolved to equal rapidities by applying the Balitsky-JIMWLK Hamiltonian $H$ [3]. The contraction of Reggeons of equal rapidity is evaluated in terms of free propagators $\left\langle W^{a}(p) \mid W^{b}(q)\right\rangle=$ $\frac{i}{p^{2}} \delta^{a b} \delta^{2-2 \epsilon}(p-q)+\mathcal{O}\left(g_{s}^{2}\right)$ [7]. To compute the amplitude

$$
\frac{i}{2 s} \frac{1}{Z_{i} Z_{j}} \mathcal{M}_{i j \rightarrow i j}=\left\langle\psi_{j}\left|e^{-H L}\right| \psi_{i}\right\rangle
$$

we expand the projectile and target in the number of Reggeons $n$, such that $\left|\psi_{i}\right\rangle=\sum_{n=1}^{\infty}\left(r_{\Gamma} \alpha_{s}\right)^{(n-1) / 2}\left|i_{n}\right\rangle$, where

$$
\left|i_{n}\right\rangle=\left|i_{n}\right\rangle^{\mathrm{LO}}+\sum_{k=1}^{\infty}\left(\frac{\alpha_{s}}{\pi}\right)^{k}\left|i_{n}\right\rangle^{\mathrm{N}^{k} \mathrm{LO}}
$$


with the tree level normalized as $\left\langle j_{1} \mid i_{1}\right\rangle=\frac{i g_{s}^{2}}{t} \mathbf{T}_{i} \cdot \mathbf{T}_{j}$. The Hamiltonian $H$ allows transitions between states with different numbers of Reggeons [7]. However, signature symmetry excludes transitions between states of odd and even numbers of Reggeons, e.g. $\left\langle j_{3}\left|e^{-H L}\right| i_{2}\right\rangle=0$. Only odd (even) transitions contribute to the odd (even) amplitude. We denote individual Hamiltonians converting an $n$ Reggeon state to a $k$ Reggeon state as $H_{n \rightarrow k}$. Defining channels of color flow [39] by

$\mathbf{T}_{s}=\mathbf{T}_{1}+\mathbf{T}_{2}, \quad \mathbf{T}_{t}=\mathbf{T}_{1}+\mathbf{T}_{4}, \quad \mathbf{T}_{u}=\mathbf{T}_{1}+\mathbf{T}_{3}$,

the single Reggeon evolves with $H_{1 \rightarrow 1}=-\mathbf{T}_{t}^{2} \alpha_{g}(t)$, with $\left\langle j_{1}\left|\mathbf{T}_{t}^{2}\right| i_{1}\right\rangle=C_{A}\left\langle j_{1} \mid i_{1}\right\rangle ;$ NNLL accuracy requires $\mathcal{O}\left(\alpha_{s}^{3}\right)$ corrections in $\alpha_{g}(t)$ of Eq. (2).

Multi-Reggeon transitions $H_{n \rightarrow k}$ were computed to leading order in [8] by expanding the Balitsky-JIMWLK Hamiltonian [3] in the Reggeon field, obtaining $[7,8]$

$$
H=\sum_{n \geq 1}\left[H_{n \rightarrow n}^{\mathrm{LO}}+\sum_{l>0}\left(\alpha_{s} r_{\Gamma}\right)^{l} H_{n \rightarrow n \pm 2 l}^{\mathrm{LO}}\right]+\ldots
$$

where the ellipsis stand for $\mathcal{O}\left(\alpha_{s}^{2}\right)$ corrections to $H$ [40]; these are not required for multi-Reggeon transitions at NNLL. To determine $\mathcal{M}^{(-, n, n-2)}$ it is convenient to extract $H_{1 \rightarrow 1}$ in (7), defining the reduced amplitude,

$$
\frac{i}{2 s} \hat{\mathcal{M}}_{i j \rightarrow i j} \equiv\left\langle\psi_{j}\left|e^{-\left(H-H_{1 \rightarrow 1}\right) L}\right| \psi_{i}\right\rangle=\left\langle\psi_{j}\left|e^{-\hat{H} X}\right| \psi_{i}\right\rangle,
$$

along with a reduced Hamiltonian $\hat{H}$ and an expansion parameter $X \equiv \frac{\alpha_{s}}{\pi} r_{\Gamma} L$. Note that $\hat{H}_{1 \rightarrow 1}=0$. Expanding (11) and collecting the NNLL in the odd amplitude we find, to all orders in $\alpha_{s}$,

$$
\begin{aligned}
& \frac{i}{2 s} \hat{\mathcal{M}}_{i j \rightarrow i j}^{(-), \mathrm{NNLL}} \\
& =\left(\frac{\alpha_{s}}{\pi}\right)^{2}\left\{r _ { \Gamma } ^ { 2 } \pi ^ { 2 } \left[\sum_{k=0}^{\infty} \frac{(-X)^{k}}{k !}\left\langle j_{3}\left|\hat{H}_{3 \rightarrow 3}^{k}\right| i_{3}\right\rangle\right.\right. \\
& \quad+\sum_{k=1}^{\infty} \frac{(-X)^{k}}{k !}\left[\left\langle j_{1}\left|\hat{H}_{3 \rightarrow 1} \hat{H}_{3 \rightarrow 3}^{k-1}\right| i_{3}\right\rangle+\left\langle j_{3}\left|\hat{H}_{3 \rightarrow 3}^{k-1} \hat{H}_{1 \rightarrow 3}\right| i_{1}\right\rangle\right] \\
& \left.\left.\quad+\sum_{k=2}^{\infty} \frac{(-X)^{k}}{k !}\left\langle j_{1}\left|\hat{H}_{3 \rightarrow 1} \hat{H}_{3 \rightarrow 3}^{k-2} \hat{H}_{1 \rightarrow 3}\right| i_{1}\right\rangle\right]^{\mathrm{LO}}+\left\langle j_{1} \mid i_{1}\right\rangle^{\mathrm{NNLO}}\right\}
\end{aligned}
$$

With the sole exception of the term $\left\langle j_{1} \mid i_{1}\right\rangle^{\mathrm{NNLO}}$, which is extracted from two-loop amplitudes [8], Eq. (12) describes the whole NNLL tower using the leading-order formalism.

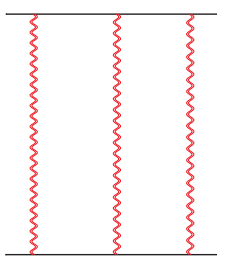

(a)

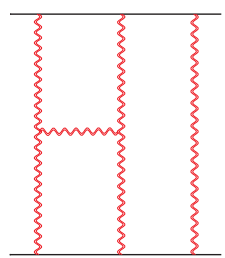

(b)

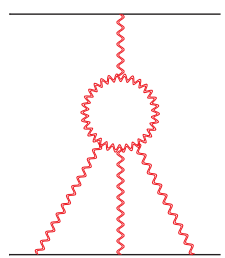

(c)
FIG. 1. Three-Reggeon exchange at two and three loops [(a) and (b)] and their mixing into a single Reggeon (c).

\section{RESULTS AT NNLL}

We proceed to evaluate $\hat{\mathcal{M}}$ in (12) to four loops, following the notation of (4).

\section{A. Two loops}

According to (12) there are two distinct contributions to the two-loop amplitude $\hat{\mathcal{M}}^{(-, 2,0)}$. The first is the single Reggeon exchange, $\left\langle j_{1} \mid i_{1}\right\rangle^{\mathrm{NNLO}}$, which may be read off Eq. (5),

$$
\left\langle j_{1} \mid i_{1}\right\rangle^{\mathrm{NNLO}}=\left(D_{i}^{(2)}+D_{j}^{(2)}+D_{i}^{(1)} D_{j}^{(1)}\right)\left\langle j_{1} \mid i_{1}\right\rangle .
$$

The second is the three Reggeon exchange depicted in Fig. 1(a) (where there is an implicit sum over permutations of the three-Reggeon attachment to the Wilson line),

$$
\begin{aligned}
\left\langle j_{3} \mid i_{3}\right\rangle & =-3\left(\frac{1}{\epsilon^{2}}-6 \epsilon f_{\epsilon}\right) \quad \mathbf{C}_{33}^{(2)}\left\langle j_{1} \mid i_{1}\right\rangle \\
f_{\epsilon} & \equiv \zeta_{3}+\frac{3}{2} \epsilon \zeta_{4}+\mathcal{O}\left(\epsilon^{2}\right) ; \quad \mathbf{C}_{33}^{(2)} \equiv \frac{1}{24}\left(\left(\mathbf{T}_{s-u}^{2}\right)^{2}-\frac{C_{A}^{2}}{12}\right),
\end{aligned}
$$

where $\mathbf{T}_{s-u}^{2} \equiv \frac{1}{2}\left(\mathbf{T}_{s}^{2}-\mathbf{T}_{u}^{2}\right)$. Adding up the two contributions we obtain $\hat{\mathcal{M}}^{(-, 2,0)}$ in agreement with [8].

\section{B. Three loops}

There are two contributions to $\hat{\mathcal{M}}^{(-, 3,1)}$ in eq. (12). The first, in Fig. 1(b), is the $\hat{H}_{3 \rightarrow 3}$ evolution,

$$
\begin{aligned}
\left\langle j_{3}\left|\hat{H}_{3 \rightarrow 3}\right| i_{3}\right\rangle & =\left[\frac{1}{\epsilon^{3}}\left(C_{A}-\frac{5}{6} \mathbf{T}_{t}^{2}\right)+2 f_{\epsilon}\right. \\
& \left.\times\left(C_{A}-\frac{41}{6} \mathbf{T}_{t}^{2}\right)+\mathcal{O}\left(\epsilon^{2}\right)\right] \mathbf{C}_{33}^{(2)}\left\langle j_{1} \mid i_{1}\right\rangle,
\end{aligned}
$$

which relates to $\mathbf{C}_{33}^{(2)}$ of Eq. (14). This relation stems from the fact that the action of $\hat{H}_{3 \rightarrow 3}$ is symmetrized under permutations of the three Reggeons, as both the target and projectile wavefunctions are symmetric.

The new type of contribution at three loops, Fig. 1(c), arises from the term involving $\hat{H}_{3 \rightarrow 1}$ in Eq. (12), 


$$
\left\langle j_{1}\left|\hat{H}_{3 \rightarrow 1}\right| i_{3}\right\rangle=\frac{1}{36}\left(-\frac{1}{\epsilon^{3}}+70 f_{\epsilon}+\mathcal{O}\left(\epsilon^{2}\right)\right) d_{i}\left\langle j_{1} \mid i_{1}\right\rangle,
$$

and a similar one involving $\hat{H}_{1 \rightarrow 3}$, obtained upon replacing $d_{i} \equiv \frac{d_{A R_{i}}}{N_{R_{i}}} \frac{1}{C_{R_{i}}}$ in (16) by $d_{j}$. Given the representation $R_{i}$ we defined respectively, $N_{R_{i}}, C_{R_{i}}$ and $d_{A R_{i}}$ as the dimension, the quadratic, and the quartic Casimirs,

$$
d_{A R_{i}} \equiv \frac{1}{6} \sum_{\sigma \in \mathcal{S}_{3}} \operatorname{tr}\left(F^{a} F^{b} F^{c} F^{d}\right) \operatorname{tr}\left(\mathbf{T}^{a} \mathbf{T}^{\sigma(b)} \mathbf{T}^{\sigma(c)} \mathbf{T}^{\sigma(d)}\right)
$$

with $\mathbf{T}^{a}$ and $F^{a}$, respectively, the generators in the $R_{i}$ and the adjoint representations. In contrast to (14)-(15), Eq. (16) does not involve a matrix in color space, but is simply proportional to $\left\langle j_{1} \mid i_{1}\right\rangle$. This is due to the fact that color is carried by a single Reggeon on either the target or projectile sides. This property holds for all terms in the second and third lines of (12) at any order [41].

\section{Four loops}

According to (12), the four-loop NNLL amplitude $\hat{\mathcal{M}}^{(-, 4,2)}$ is a sum of four terms,

$$
\begin{aligned}
\frac{i}{2 s} \hat{\mathcal{M}}^{(-, 4,2)}= & \frac{r_{\Gamma}^{4} \pi^{2}}{2}\left[\left\langle j_{1}\left|\hat{H}_{3 \rightarrow 1} \hat{H}_{1 \rightarrow 3}\right| i_{1}\right\rangle+\left\langle j_{3}\left|\hat{H}_{3 \rightarrow 3}^{2}\right| i_{3}\right\rangle\right. \\
& \left.+\left\langle j_{1}\left|\hat{H}_{3 \rightarrow 1} \hat{H}_{3 \rightarrow 3}\right| i_{3}\right\rangle+\left\langle j_{3}\left|\hat{H}_{3 \rightarrow 3} \hat{H}_{1 \rightarrow 3}\right| i_{1}\right\rangle\right] .
\end{aligned}
$$

Their calculation is the main result of this paper. Despite the high loop order, the integration in transverse-momentum space is relatively straightforward; all integrals depend on a single scale, $t$, and involve up to four propagators in a loop, and they can be performed [42] using known techniques [43]. In turn, expressing the color structure of Eq. (17) in an operator form, valid for general representations, requires new techniques [42]. The first term, Fig. 2(a), yields

$$
\begin{aligned}
\left\langle j_{1}\left|\hat{H}_{3 \rightarrow 1} \hat{H}_{1 \rightarrow 3}\right| i_{1}\right\rangle= & \frac{1}{432}\left[-\left(\frac{C_{A}^{4}}{12}+\frac{d_{A A}}{N_{A}}\right) \frac{1}{\epsilon^{4}}\right. \\
& \left.+\left(\frac{101}{6} C_{A}^{4}+220 \frac{d_{A A}}{N_{A}}\right) \frac{f_{\epsilon}}{\epsilon}+\mathcal{O}(\epsilon)\right] \\
& \times\left\langle j_{1} \mid i_{1}\right\rangle,
\end{aligned}
$$

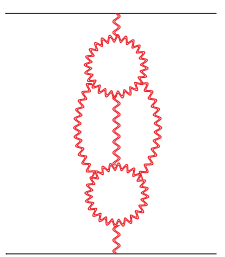

(a)

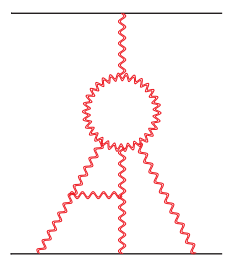

(b)

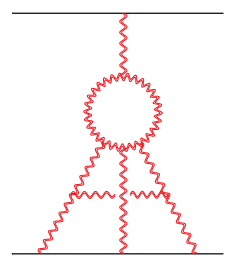

(c)
FIG. 2. Four-loop mixing between a single and three Reggeons states through $\hat{H}_{1 \rightarrow 3}$ and/or $\hat{H}_{3 \rightarrow 1}$.

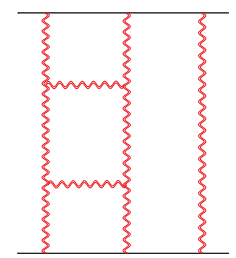

(a)

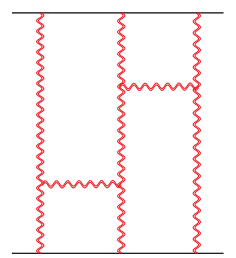

(b)
FIG. 3. Four-loop evolution of the three Reggeon state with two insertions of the $\hat{H}_{3 \rightarrow 3}$ Hamiltonian.

involving the quartic Casimir in the adjoint representation, $d_{A A}$.

The second term in (17) contains a repeated application of the $\hat{H}_{3 \rightarrow 3}$ Hamiltonian, yielding two independent color structures, Fig. 3, each multiplying a different integral. This breaks the permutation symmetry of the three-Reggeon wavefunction which was responsible for the simple structure in (15), where $\mathbf{T}_{s-u}^{2}$ entered solely via $\mathbf{C}_{33}^{(2)}$. The resulting color structure is thus more complex

$$
\begin{aligned}
\left\langle j_{3}\left|\hat{H}_{3 \rightarrow 3}^{2}\right| i_{3}\right\rangle= & \frac{1}{144}\left[\frac{\mathbf{C}_{33}^{(4,-4)}}{\epsilon^{4}}+\frac{2 f_{\epsilon}}{\epsilon} \mathbf{C}_{33}^{(4,-1)}+\mathcal{O}(\epsilon)\right]\left\langle j_{1} \mid i_{1}\right\rangle \\
\mathbf{C}_{33}^{(4,-4)}= & 6\left(17 C_{A} \mathbf{T}_{t}^{2}-6 C_{A}^{2}-6\left(\mathbf{T}_{t}^{2}\right)^{2}\right) \mathbf{C}_{33}^{(2)} \\
& -\frac{3}{4} \mathbf{T}_{s-u}^{2}\left(\mathbf{T}_{t}^{2}\right)^{2} \mathbf{T}_{s-u}^{2}+\frac{25}{144} C_{A}^{4}+\frac{1}{3} \frac{d_{A A}}{N_{A}} \\
& -3 C_{A}\left(d_{i}+d_{j}\right) \\
\mathbf{C}_{33}^{(4,-1)}= & 18\left(521 C_{A} \mathbf{T}_{t}^{2}-300 C_{A}^{2}-220\left(\mathbf{T}_{t}^{2}\right)^{2}\right) \mathbf{C}_{33}^{(2)} \\
& -101 \mathbf{C}_{33}^{(4,-4)}
\end{aligned}
$$

involving an additional term, $\mathbf{T}_{s-u}^{2}\left(\mathbf{T}_{t}^{2}\right)^{2} \mathbf{T}_{s-u}^{2}$.

The third term in (17) gives rise to two distinct color structures, Figs. 2(b) and 2(c), depending on which Reggeons are acted upon by $\hat{H}_{3 \rightarrow 3}$. As at three loops in (16), the result is proportional to the tree-level amplitude, specifically, Fig. 2(c) vanishes, while Fig. 2(b) yields

$$
\left\langle j_{1}\left|\hat{H}_{3 \rightarrow 1} \hat{H}_{3 \rightarrow 3}\right| i_{3}\right\rangle=\frac{C_{A} d_{i}}{144}\left[\frac{1}{\epsilon^{4}}-208 \frac{f_{\epsilon}}{\epsilon}+\mathcal{O}(\epsilon)\right]\left\langle j_{1} \mid i_{1}\right\rangle .
$$

Finally, the fourth term in (17) can be simply obtained from (20) upon replacing $d_{i}$ by $d_{j}$.

Remarkably, $f_{\epsilon}$ of (14) features at three loops in (15)-(16) and four loops in (18)-(20), all manifesting the simple relation between the coefficients of transcendental weights three and four (this does not extend to weight five). Adding up the four contributions to (17), the four-loop NNLL reduced amplitude is found to be 


$$
\begin{aligned}
\hat{\mathcal{M}}^{(-, 4,2)} & =\frac{r_{\Gamma}^{4} \pi^{2}}{144}\left[\mathbf{C}_{\mathcal{M}}^{(-4)} \frac{1}{\epsilon^{4}}+\mathbf{C}_{\mathcal{M}}^{(-1)} \frac{f_{\epsilon}}{\epsilon}+\mathcal{O}(\epsilon)\right] \mathcal{M}^{\text {tree }} \\
\mathbf{C}_{\mathcal{M}}^{(-4)} & =\frac{1}{2} \mathbf{C}_{33}^{(4,-4)}-\frac{C_{A}^{4}}{72}-\frac{1}{6} \frac{d_{A A}}{N_{A}}+\frac{1}{2} C_{A}\left(d_{i}+d_{j}\right) \\
\mathbf{C}_{\mathcal{M}}^{(-1)} & =\mathbf{C}_{33}^{(4,-1)}+\frac{101 C_{A}^{4}}{36}+\frac{110}{3} \frac{d_{A A}}{N_{A}}-104 C_{A}\left(d_{i}+d_{j}\right) .
\end{aligned}
$$

Interestingly, while the separate contributions have planar components, we find that $\hat{\mathcal{M}}^{(-, 4,2)}$ is nonplanar.

\section{INFRARED DIVERGENCES}

It has long been recognized that the exponentiation of high-energy logarithms is interlinked with that of infrared singularities $[7,16,34,36,38]$ and the interplay between the two has been instrumental $[8-11,18]$. We now take another step in this direction; comparing the NNLL amplitude with the known infrared structure provides an independent check of all multiple poles in $\epsilon$, while the single pole allows us to extract the four-loop soft anomalous dimension at NNLL.

It is well known that infrared divergences in amplitudes factorize and exponentiate $[31,44]$ according to

$$
\mathcal{M}=\mathbf{Z} \cdot \mathcal{H}, \quad \mathbf{Z}=\mathcal{P} \exp \left\{-\frac{1}{2} \int_{0}^{\mu^{2}} \frac{d \lambda^{2}}{\lambda^{2}} \boldsymbol{\Gamma}\right\}
$$

where $\mathcal{H}$ is an infrared-renormalized hard amplitude, which is finite, and $\boldsymbol{\Gamma}$ is the soft anomalous dimension. In the highenergy limit the latter takes the form [16]

$$
\boldsymbol{\Gamma}=\frac{\gamma_{K}}{2}\left[L \mathbf{T}_{t}^{2}+i \pi \mathbf{T}_{s-u}^{2}\right]+\Gamma_{i}+\Gamma_{j}+\boldsymbol{\Delta},
$$

where $\Gamma_{i}$ is defined in (6) and $\Delta$ represents nondipole corrections starting at three loops [31,45]. The dipole contribution is well known [16,34]; the $L \mathbf{T}_{t}^{2}$ term contributes starting at LL, while the $i \pi \mathbf{T}_{s-u}^{2}$ and $\Gamma_{i}$ terms start at NLL. Here we focus on $\Delta$, which we expand as

$$
\Delta=\sum_{n=3}^{\infty}\left(\frac{\alpha_{s}}{\pi}\right)^{n} \sum_{m=0}^{n-1} L^{m} \Delta^{(n, m)} .
$$

The NLL tower $\boldsymbol{\Delta}^{(n, n-1)}$ is purely imaginary, and was recently obtained to all orders [9] from the two-Reggeon amplitude. It starts contributing at four loops [7], where

$$
\boldsymbol{\Delta}^{(4,3)}=-i \pi \frac{\zeta_{3}}{24}\left[\mathbf{T}_{t}^{2},\left[\mathbf{T}_{t}^{2}, \mathbf{T}_{s-u}^{2}\right]\right] \mathbf{T}_{t}^{2}
$$

The NNLL tower $\boldsymbol{\Delta}^{(n, n-2)}$ starts at three loops, with a purely imaginary contribution,

$$
\boldsymbol{\Delta}^{(3,1)}=i \pi \frac{\zeta_{3}}{4}\left[\mathbf{T}_{t}^{2},\left[\mathbf{T}_{t}^{2}, \mathbf{T}_{s-u}^{2}\right]\right],
$$

determined in [8] on the basis of the three-loop calculation of $\boldsymbol{\Delta}$ in general kinematics [45]. Thanks to the present calculation of the four-loop amplitude, we obtain the first nontrivial real contribution to this tower, $\operatorname{Re}\left[\boldsymbol{\Delta}^{(4,2)}\right]$. To this end we invert (22), and use (7) and (11) to restore the Regge trajectory, obtaining

$$
\mathcal{H}=Z_{i} Z_{j} \mathbf{Z}^{-1} e^{\alpha_{g} \mathbf{T}_{t}^{2} L} \hat{\mathcal{M}}
$$

The right-hand side of (27) at NNLL depends on $D_{i}^{(k)}$ in (13) and on two- and three-loop Regge-trajectory coefficients, which are all theory specific. However, the poles in $\hat{\mathcal{M}}$ are universal [42], so upon requiring that the hard function $\mathcal{H}^{(4,2)}$ is finite we verify $\mathbf{C}_{\mathcal{M}}^{(-4)}$ in (21) and get

$$
\operatorname{Re}\left[\boldsymbol{\Delta}^{(4,2)}\right]=\zeta_{2} \zeta_{3} \mathbf{C}_{\Delta}^{(4,2)},
$$

where the color structure can be written compactly as

$$
\begin{aligned}
\mathbf{C}_{\Delta}^{(4,2)}= & \frac{1}{4} \mathbf{T}_{t}^{2}\left[\mathbf{T}_{t}^{2},\left(\mathbf{T}_{s-u}^{2}\right)^{2}\right]+\frac{3}{4}\left[\mathbf{T}_{s-u}^{2}, \mathbf{T}_{t}^{2}\right] \mathbf{T}_{t}^{2} \mathbf{T}_{s-u}^{2} \\
& +\frac{d_{A A}}{N_{A}}-\frac{C_{A}^{4}}{24} .
\end{aligned}
$$

As expected, $\operatorname{Re}\left[\boldsymbol{\Delta}^{(4,2)}\right]$ is nonplanar. This result holds for any gauge theory.

Equations (21) and (27) can be further used to determine the finite terms in the hard function at four loops. We can deduce from the known Regge trajectory $[8,17,29]$ that in $\mathcal{N}=4$ super-Yang-Mills we have

$$
\operatorname{Re}\left[\mathcal{H}_{\mathcal{N}=4}^{(4,2)}\right]=\left[\frac{C_{A}^{4}}{128} \zeta_{3}^{2}+\frac{3}{16} \zeta_{4} \zeta_{2} \mathbf{C}_{\Delta}^{(4,2)}\right] \mathcal{M}^{\text {tree }}
$$

The planar limit, $N_{c} \rightarrow \infty$, is already known [46]; the nonplanar correction, the second term, is a new result.

\section{CONCLUSIONS}

In this paper we demonstrated that the entire signatureodd tower of NNLL contributions to partonic $2 \rightarrow 2$ amplitudes in the Regge limit, is computable in full color using an iterated solution of the rapidity evolution equation. Only the leading-order Hamiltonian is required in this iteration, implying a remarkable universality of these corrections across all gauge theories starting from three loops. We showed that the result, given by Eq. (12), has a simple diagrammatic interpretation as a class of ladder graphs involving three Reggons with any number of rungs. This is a step towards a formulation of an interacting Reggeon field theory. 
In this framework we performed an explicit computation of the (reduced) signature-odd $2 \rightarrow 2$ amplitude to four loops through finite terms, obtaining new results at NNLL accuracy, valid for general color representations. Interestingly, the four-loop result, Eq. (21), is entirely nonplanar. It involves quartic Casimirs associated with the representations of the scattered partons as well as a purely adjoint one. We observe an interesting relation between the transcendental weight 3 and 4 contributions, already visible at two loops (14). This relation holds also at three loops, and at four loops it amounts to a relation between the $1 / \epsilon$ pole term and the finite terms.

Matching the result to the known exponentiation properties of soft singularities provides a powerful check on the computation, and allows us to extract the four-loop NNLL soft anomalous dimension, presented in (28). We also determine the finite terms of the $\mathcal{N}=4$ amplitude, Eq. (30). Regge limit results have proven instrumental in bootstrapping the three-loop soft anomalous dimension in general kinematics [18]. Our result paves the way for a four-loop bootstrap. Reference [32] analyzed the color structure of the soft anomalous dimension, incorporating the recently computed four-loop cusp anomalous dimension $[47,48]$, which introduces quartic Casimirs. Our results show that the soft anomalous dimension contains additional quartic Casimirs, beyond those associated with the cusp.

\section{ACKNOWLEDGMENTS}

We would like to thank Simon Caron-Huot for insightful comments and Niamh Maher for discussions regarding the color structure. E. G. and G. F. are supported by the STFC Consolidated Grant 'Particle Physics at the Higgs Centre'. G. F. is supported by the ERC Starting Grant No. 715049 'QCDforfuture'. C. M.'s work is supported by the Italian Ministry of University and Research (MIUR), Grant No. PRIN 20172LNEEZ. L. V, is supported by the Fellini Fellowship for Innovation at INFN, funded by the European Union's Horizon 2020 research programme under the Marie Skłodowska-Curie Cofund Action, Grant Agreement No. 754496.
[1] E. A. Kuraev, L. N. Lipatov, and V. S. Fadin, Zh. Eksp. Teor. Fiz. 72, 377 (1977); [Sov. Phys. JETP 45, 199 (1977)].

[2] I. I. Balitsky and L. N. Lipatov, Yad. Fiz. 28, 1597 (1978) [Sov. J. Nucl. Phys. 28, 822 (1978)].

[3] I. Balitsky, Nucl. Phys. B463, 99 (1996); Y. V. Kovchegov, Phys. Rev. D 60, 034008 (1999); J. Jalilian-Marian, A. Kovner, L. D. McLerran, and H. Weigert, Phys. Rev. D 55, 5414 (1997); J. Jalilian-Marian, A. Kovner, A. Leonidov, and H. Weigert, Phys. Rev. D 59, 014014 (1998); E. Iancu, A. Leonidov, and L. D. McLerran, Phys. Lett. B 510, 133 (2001).

[4] P. D. B. Collins, An Introduction to Regge Theory and HighEnergy Physics, Cambridge Monographs on Mathematical Physics (Cambridge University Press, Cambridge, England, 2009).

[5] L. J. Dixon, C. Duhr, and J. Pennington, J. High Energy Phys. 10 (2012) 074.

[6] L. J. Dixon, J. M. Drummond, C. Duhr, and J. Pennington, J. High Energy Phys. 06 (2014) 116.

[7] S. Caron-Huot, J. High Energy Phys. 05 (2015) 093.

[8] S. Caron-Huot, E. Gardi, and L. Vernazza, J. High Energy Phys. 06 (2017) 016.

[9] S. Caron-Huot, E. Gardi, J. Reichel, and L. Vernazza, J. High Energy Phys. 03 (2018) 098.

[10] S. Caron-Huot, E. Gardi, J. Reichel, and L. Vernazza, J. High Energy Phys. 08 (2020) 116.

[11] E. Gardi, S. Caron-Huot, J. Reichel, and L. Vernazza, Proc. Sci., RADCOR2019 (2019) 050 [arXiv:1912.10883].
[12] V. Del Duca, S. Druc, J. Drummond, C. Duhr, F. Dulat, R. Marzucca, G. Papathanasiou, and B. Verbeek, Phys. Rev. Lett. 124, 161602 (2020).

[13] J. Bartels, arXiv:2005.08818.

[14] S. Caron-Huot, L. J. Dixon, A. McLeod, and M. von Hippel, Phys. Rev. Lett. 117, 241601 (2016).

[15] S. Caron-Huot, L. J. Dixon, F. Dulat, M. von Hippel, A. J. McLeod, and G. Papathanasiou, J. High Energy Phys. 08 (2019) 016.

[16] V. Del Duca, C. Duhr, E. Gardi, L. Magnea, and C. D. White, J. High Energy Phys. 12 (2011) 021.

[17] J. M. Henn and B. Mistlberger, Phys. Rev. Lett. 117, 171601 (2016).

[18] O. Almelid, C. Duhr, E. Gardi, A. McLeod, and C. D. White, J. High Energy Phys. 09 (2017) 073.

[19] L. Lipatov, Sov. J. Nucl. Phys. 23, 338 (1976).

[20] E. A. Kuraev, L. N. Lipatov, and V. S. Fadin, Sov. Phys. JETP 44, 443 (1976).

[21] F. E. Low, Phys. Rev. D 12, 163 (1975).

[22] S. Nussinov, Phys. Rev. Lett. 34, 1286 (1975).

[23] J. F. Gunion and D.E. Soper, Phys. Rev. D 15, 2617 (1977).

[24] L. N. Lipatov, JETP Lett. 59, 596 (1994).

[25] L. D. Faddeev and G. P. Korchemsky, Phys. Lett. B 342, 311 (1995).

[26] S. E. Derkachov, G. P. Korchemsky, and A. N. Manashov, Nucl. Phys. B617, 375 (2001).

[27] S. E. Derkachov, G. P. Korchemsky, J. Kotanski, and A. N. Manashov, Nucl. Phys. B645, 237 (2002). 
[28] V. Fadin, R. Fiore, M. Kozlov, and A. Reznichenko, Phys. Lett. B 639, 74 (2006); V. Fadin, M. Kozlov, and A. Reznichenko, Phys. Rev. D 92, 085044 (2015).

[29] V. S. Fadin, M. Kotsky, and R. Fiore, Phys. Lett. B 359, 181 (1995); V. S. Fadin, R. Fiore, and M. Kotsky, Phys. Lett. B 387, 593 (1996); V. S. Fadin, R. Fiore, and A. Quartarolo, Phys. Rev. D 53, 2729 (1996); J. Blumlein, V. Ravindran, and W. van Neerven, Phys. Rev. D 58, 091502 (1998).

[30] G. Korchemsky and A. Radyushkin, Nucl. Phys. B283, 342 (1987).

[31] E. Gardi and L. Magnea, J. High Energy Phys. 03 (2009) 079; Frascati Phys. Ser. 50, 137 (2010).T. Becher and M. Neubert, Phys. Rev. Lett. 102, 162001 (2009); 111, 199905 (E) (2013).

[32] T. Becher and M. Neubert, J. High Energy Phys. 01 (2020) 025.

[33] S. Moch, J. A. M. Vermaseren, and A. Vogt, Phys. Lett. B 625, 245 (2005).

[34] V. Del Duca, G. Falcioni, L. Magnea, and L. Vernazza, J. High Energy Phys. 02 (2015) 029.

[35] V. Del Duca and E. Glover, J. High Energy Phys. 10 (2001) 035 .

[36] V. Del Duca, G. Falcioni, L. Magnea, and L. Vernazza, Phys. Lett. B 732, 233 (2014).

[37] V. Fadin, AIP Conf. Proc. 1819, 060003 (2017); V. Fadin and L. Lipatov, Eur. Phys. J. C 78, 439 (2018).

[38] I. A. Korchemskaya and G. P. Korchemsky, Nucl. Phys. B437, 127 (1995); Phys. Lett. B 387, 346 (1996).

[39] Y. Dokshitzer and G. Marchesini, Phys. Lett. B 631, 118 (2005).
[40] I. Balitsky and A. V. Belitsky, Nucl. Phys. B629, 290 (2002); I. Balitsky and G. A. Chirilli, Phys. Rev. D 77, 014019 (2008); E. Gardi, J. Kuokkanen, K. Rummukainen, and H. Weigert, Nucl. Phys. A784, 282 (2007); I. Balitsky and G. A. Chirilli, Phys. Rev. D 88, 111501 (2013); A. Kovner, M. Lublinsky, and Y. Mulian, Phys. Rev. D 89, 061704 (2014).

[41] We thank Simon Caron-Huot for this important observation.

[42] G. Falcioni, E. Gardi, N. Maher, C. Milloy, and L. Vernazza, Scattering amplitudes in the Regge limit and the soft anomalous dimension through four loops (to be published).

[43] R. N. Lee, J. Phys. Conf. Ser. 523, 012059 (2014); B. Ruijl, T. Ueda, and J. Vermaseren, Comput. Phys. Commun. 253, 107198 (2020).

[44] S. Catani, Phys. Lett. B 427, 161 (1998); G. F. Sterman and M. E. Tejeda-Yeomans, Phys. Lett. B 552, 48 (2003); S. Aybat, L. J. Dixon, and G. F. Sterman, Phys. Rev. Lett. 97, 072001 (2006); Phys. Rev. D 74, 074004 (2006).

[45] O. Almelid, C. Duhr, and E. Gardi, Phys. Rev. Lett. 117, 172002 (2016).

[46] C. Anastasiou, Z. Bern, L. J. Dixon, and D. Kosower, Phys. Rev. Lett. 91, 251602 (2003); Z. Bern, L. J. Dixon, and V. A. Smirnov, Phys. Rev. D 72, 085001 (2005); J. Drummond, G. Korchemsky, and E. Sokatchev, Nucl. Phys. B795, 385 (2008).

[47] J. M. Henn, G. P. Korchemsky, and B. Mistlberger, J. High Energy Phys. 04 (2020) 018.

[48] A. von Manteuffel, E. Panzer, and R. M. Schabinger, Phys. Rev. Lett. 124, 162001 (2020). 